\title{
Discovery of X-ray absorption features from the dipping low-mass X-ray binary XB 1916-053 with XMM-Newton
}

\author{
L. Boirin ${ }^{1}$, A. N. Parmar ${ }^{1}$, D. Barret ${ }^{2}$, S. Paltani ${ }^{3}$, and J. E. Grindlay ${ }^{4}$ \\ 1 Astrophysics Missions Division, Research and Scientific Support Department of ESA, ESTEC, Postbus 299, \\ 2200 AG Noordwijk, The Netherlands \\ 2 Centre d'Etude Spatiale des Rayonnements, CNRS/UPS, 9 Av. du Colonel Roche, 31028 Toulouse Cedex 4, France \\ 3 Laboratoire d'Astrophysique de Marseille, Traverse du Siphon, BP 8, 13376 Marseille Cedex 12, France \\ ${ }^{4}$ Harvard-Smithsonian Center for Astrophysics, 60 Garden Street Cambridge, MA 02138, USA
}

Received 21 October 2003 / Accepted 3 February 2004

\begin{abstract}
We report the discovery of narrow Fe XXV and Fe XXVI K $\alpha$ X-ray absorption lines at $6.65_{-0.02}^{+0.05}$ and $6.95_{-0.04}^{+0.05} \mathrm{keV}$ in the persistent emission of the dipping low-mass X-ray binary (LMXB) XB 1916-053 during an XMM-Newton observation performed in September 2002. In addition, there is marginal evidence for absorption features at $1.48 \mathrm{keV}, 2.67 \mathrm{keV}, 7.82 \mathrm{keV}$ and $8.29 \mathrm{keV}$ consistent with Mg XII, S XVI, Ni XXVII K $\alpha$ and Fe XXVI K $\beta$ transitions, respectively. Such absorption lines from highly ionized ions are now observed in a number of high inclination (i.e. close to edge-on) LMXBs, such as XB 1916-053, where the inclination is estimated to be between $60-80^{\circ}$. This, together with the lack of any orbital phase dependence of the features (except during dips), suggests that the highly ionized plasma responsible for the absorption lines is located in a cylindrical geometry around the compact object. Using the ratio of Fe XXV and Fe XXVI column densities, we estimate the photo-ionization parameter of the absorbing material, $\xi$, to be $10^{3.92} \mathrm{erg} \mathrm{cm} \mathrm{s}^{-1}$. Only the Fe XXV line is observed during dipping intervals and the upper-limits to the Fe XXVI column density are consistent with a decrease in the amount of ionization during dipping intervals. This implies the presence of cooler material in the line of sight during dipping. We also report the discovery of a $0.98 \mathrm{keV}$ absorption edge in the persistent emission spectrum. The edge energy decreases to $0.87 \mathrm{keV}$ during deep dipping intervals. The detected feature may result from edges of moderately ionized $\mathrm{Ne}$ and/or Fe with the average ionization level decreasing from persistent emission to deep dipping. This is again consistent with the presence of cooler material in the line of sight during dipping.
\end{abstract}

Key words. accretion, accretion disks - stars: individual: XB 1916-053 - X-rays: binaries

\section{Introduction}

XB 1916-053 is a LMXB showing periodic intensity dips in its light curve (Walter et al. 1982; White \& Swank 1982). Dips are believed to be due to obscuration of the central X-ray source by a vertical structure located at the outer edge of the accretion disk and resulting from the impact of the accretion flow from the companion star into the disk (White \& Swank 1982). The presence of dips in XB 1916-053 and the lack of $\mathrm{X}$-ray eclipses from the companion star indicate that the system is viewed relatively close to edge-on, at an inclination angle in the range $\sim 60-80^{\circ}$ (Frank et al. 1987; Smale et al. 1988). The X-ray dip period is 50 min (e.g., White \& Swank 1982), the shortest amongst the dipping sources. The optical counterpart of XB 1916-053 shows a modulation with a period $\sim 1 \%$ longer than the X-ray dip period (e.g., Callanan et al. 1995). This discrepancy has led to several interpretations including superhumps (Schmidtke 1988) and a hierarchical triple system

Send offprint requests to: L. Boirin, e-mail: L.Boirin@sron.nl model (Grindlay et al. 1988). Retter et al. (2002) recently favored the superhump model, which invokes a precessing accretion disk, and which identifies the X-ray period as orbital. XB 1916-053 is a type I X-ray burster (Becker et al. 1977), indicating that the compact object is a neutron star. XB 1916-053 has shown quasi-periodic oscillations at various frequencies ranging from $\sim 0.2$ to $1300 \mathrm{~Hz}$ (Boirin et al. 2000), and a $270 \mathrm{~Hz}$ highly coherent oscillation during an X-ray burst (Galloway et al. 2001).

XB 1916-053, as most dippers, shows spectral hardening during dipping. However, the spectral evolution is not consistent with a simple increase of photo-electric absorption by cool material, as an excess of photons is present at low energy. Two approaches have been proposed for modelling the spectral evolution during dips. In the "absorbed plus unabsorbed" approach (e.g., Parmar et al. 1986), the persistent (non-dipping) model is used to fit the intensity-selected dip spectra, but is divided into two parts. One part is allowed to be absorbed, whereas the other one is not. The spectral evolution during dipping is 
well accounted for by a large increase in the column density of the absorbed component, and a decrease of the normalization of the unabsorbed component. The latter decrease has been attributed to effects of electron scattering in the absorber. In the "progressive covering", or "two-component" or "complex continuum" approach (e.g., Church et al. 1997), the X-ray emission originates from two components. The first one, generally modelled as a blackbody, is from a point-like region, such as a boundary layer around the compact object. The second component, generally modelled as a power-law, comes from an extended region, such as an accretion disk corona. The complex continuum approach allows both the persistent and the various intensity-selected dipping spectra to be modelled with the same two components, and explains the spectral changes by allowing partial and progressive covering of the extended source by an opaque absorber that occults various fractions of the source. The absorption applied to the point-source component is allowed to vary independently of the absorption applied to the extended component, but no partial covering is needed as the source is supposed to be point-like and thus fully covered by the absorber in the line of sight during the dips. Both approaches have been applied to XB 1916-053 (e.g., Yoshida et al. 1995; Church et al. 1997, respectively).

In addition to the continuum emission, a broad emission feature interpreted as fluorescent line emission from neutral Fe was detected from XB 1916-053, at $5.60_{-0.43}^{+0.53} \mathrm{keV}$ (Smale et al. 1992), 6.14 $4_{-1.07}^{+0.18} \mathrm{keV}$ (Bloser et al. 2000) and 5.9 $9_{-0.1}^{+0.2} \mathrm{keV}$ (Asai et al. 2000). The low energy of the emission lines compared to the $6.4 \mathrm{keV}$ expected for neutral Fe led Parmar et al. (2002) to propose that the broad emission feature was modulated with $\sim 7 \mathrm{keV}$ absorption features, which were not included in the spectral model, reducing the measured energy of the emission feature. Narrow absorption features from highly ionized Fe and other metals are now observed in a growing number of LMXBs (see Table 5), thanks to the improved sensitivity and spectral resolution offered by the new generation of instruments on-board of Chandra and XMM-Newton. These discoveries indicate that a highly ionized plasma is present in these systems, that was so far not taken into account in models. Therefore, the study of these lines appears to be extremely important to characterize the geometry and physical properties of this plasma, that could be a common property of accreting systems. The presence of absorption lines could be related to the viewing angle of the system.

In this paper, we report the discovery of narrow Fe XXV and Fe XXVI K $\alpha$ X-ray absorption lines near $7 \mathrm{keV}$ in the dipping LMXB XB 1916-053. Absorption features due to highly ionized $\mathrm{Mg}, \mathrm{S}$ and $\mathrm{Ni}$ may also be present. The Fe XXV line is also observed during dipping intervals. We also report the discovery of an absorption edge at an energy of $0.98 \mathrm{keV}$ and $0.87 \mathrm{keV}$ during the persistent and dipping intervals, respectively. Detailed modelling of the continuum emission, comparison of the "absorbed plus unabsorbed" and "complex continuum" approaches, and discussion of their physical interpretations are the subject of a separate paper (Webb et al., in preparation), and are therefore not presented here. In this paper, we focus on the X-ray absorption features, and, in order to extract their properties, we fit the continuum with a simple model and follow the complex continuum approach.

\section{Observation and data reduction}

The XMM-Newton Observatory (Jansen et al. 2001) includes three $1500 \mathrm{~cm}^{2} \mathrm{X}$-ray telescopes each with an European Photon Imaging Camera (EPIC, 0.1-15 keV) at the focus. Two of the EPIC imaging spectrometers use MOS CCDs (Turner et al. 2001) and one uses pn CCDs (Strüder et al. 2001). Reflection Grating Spectrometers (RGS, 0.35-2.5 keV, den Herder et al. 2001) are located behind two of the telescopes. XB 1916-053 was observed by XMM-Newton for $17 \mathrm{ks}$ on September 25, 2002, from 03:55 to 08:31 UTC. All the EPIC cameras were operating in timing mode, with medium thickness optical blocking filters applied. As the pn CCD is more sensitive to the presence of lines than the MOS CCDs with an effective area a factor 5 higher at $\sim 7 \mathrm{keV}$ and a better energy resolution, we concentrate on the analysis of pn data. We also carry out a spectral analysis of RGS data.

We used the X-ray data products generated by the Pipeline Processing Subsystem on October 2002. We further filtered these products and generated lightcurves and spectra using the Science Analysis Software (SAS) version 5.4.1. Electronic noise and hot or flickering pixels were rejected. No high background intervals were present in the data.

In pn timing mode, only one pn CCD chip (corresponding to a field of view of $13.6 \times 4.4)$ is used and the data from that chip are collapsed into a one-dimensional row (4.4) to be read out at high speed, the second dimension being replaced by timing information. This allows a time resolution of $30 \mu \mathrm{s}$, and photon pile-up occurs only for count rates above 1500 counts $\mathrm{s}^{-1}$. We selected only single and double events (patterns 0 to 4 ), and extracted source events from a 53" wide column centered on the source position. Background events were obtained from a column of the same width, but centered 115" from XB 1916-053. We used the latest response matrix file for the pn timing mode provided by the XMM-Newton calibration team (epn_ti40_sdY9.rsp, released in 2003 January). We generated an ancillary response file using the ftools arfgen. In the source spectrum, we observe two strong emission features near 0.25 and $0.45 \mathrm{keV}$ which are not removed by spectral modelling. Such deviations have been reported in the pn timing mode spectrum of XTE J1751-305 by Miller et al. (2003), who suggest that these are due to incorrect modelling of the instrumental response. To check this, we have downloaded several data sets from the XMM-Newton archives and extracted pn timing mode spectra for several X-ray binaries. For all of them, we find features below $0.6 \mathrm{keV}$ similar to those observed in the spectrum of XB 1916-053. Thus, we conclude that these features have an instrumental origin. In the inspected spectra, we also note deviations to the continuum fit above $10 \mathrm{keV}$. We therefore examine pn timing mode data in the 0.6-10 keV energy range. The EPIC pn spectra were rebinned to oversample the full width at half maximum of the energy resolution by a factor 3 , and to have a minimum of 20 counts per bin to allow the use of the $\chi^{2}$ statistic. In order to account for 


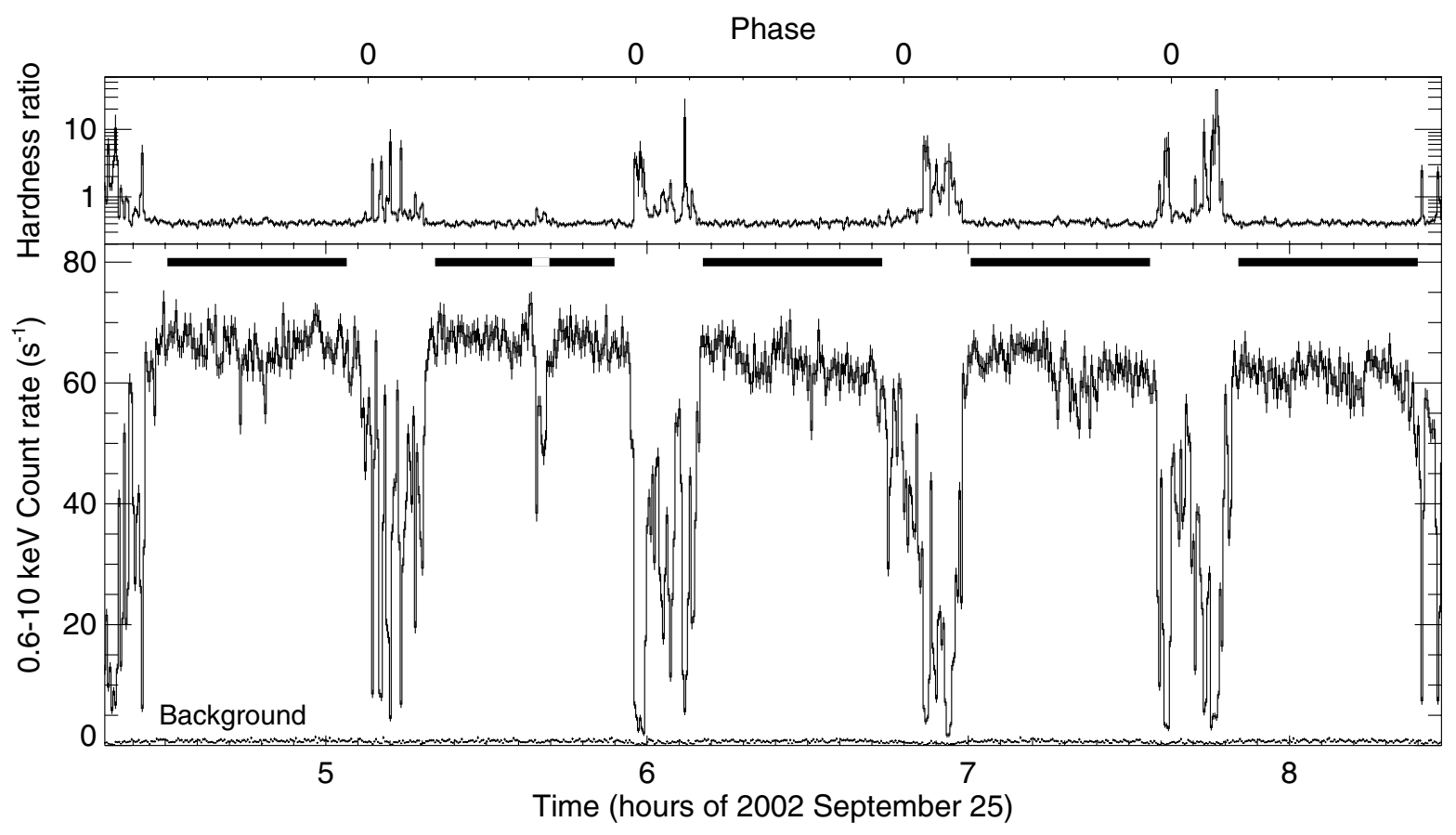

Fig. 1. 0.6-10 keV EPIC pn background-subtracted light curve of XB 1916-053 on September 25, 2002, with a binning of $20 \mathrm{~s}$ (lower panel) showing parts of 6 deep dips and weaker intermediate dipping activity. Times are barycenter-corrected. The $0.6-10 \mathrm{keV}$ background light curve taken from a region adjacent to the source is also shown. The upper panel shows the hardness ratio (counts in the $2.5-10 \mathrm{keV}$ band divided by counts in the $0.6-2.5 \mathrm{keV}$ band) with a logarithmic scale, and a binning of $20 \mathrm{~s}$. The phase according to the ephemeris of Chou et al. (2001) is indicated on the top axis. The thick horizontal lines indicate the intervals used for the persistent emission spectral analysis, corresponding to phases $0.25-0.92$, and excluding the secondary dipping activity near $5.7 \mathrm{~h}$.

systematic effects a $2 \%$ uncertainty was added quadratically to each spectral bin.

The SAS task rgsproc was used to produce calibrated RGS event lists, spectra, and response matrices. The RGS spectra were examined in the $0.5-2.0 \mathrm{keV}$ range, where the source count rate is high compared to the background count rate. The spectra were rebinned to have a minimum of 20 counts per bin to allow the use of the $\chi^{2}$ statistic.

Modelling of EPIC and RGS spectra was carried out using XSPEC version 11.2. The photo-electric absorption cross sections of Morrison \& McCammon (1983) are used throughout. All spectral uncertainties are given at $90 \%$ confidence, and upper limits at $95 \%$ confidence.

\section{Results}

\subsection{X-ray lightcurve}

The $0.6-10 \mathrm{keV}$ background-subtracted light curve obtained from the EPIC pn is shown in Fig. 1 (bottom panel) with a binning of $20 \mathrm{~s}$. The upper panel shows the hardness ratio (counts in the $2.5-10 \mathrm{keV}$ band divided by counts in the $0.6-2.5 \mathrm{keV}$ band). Times were barycenter-corrected using the SAS task barycen. The X-ray phase is indicated on the top horizontal axis of Fig. 1, and computed using MJD 50123.00944 as zero-phase epoch and an X-ray folding period of $3000.6508 \mathrm{~s}$. This X-ray ephemeris was obtained by Chou et al. (2001) from a timing analysis of RXTE observations carried out between February and October 1996 combined with historical X-ray data obtained from 1976 to 1996. Dip centers are expected at phase 0 .

No X-ray burst was observed during the $\sim 4 \mathrm{~h}$ XMM-Newton observation. Four complete dips are visible, as well as parts of dips at the start and at the end of the observation. Dip centers occur at phase $\sim 0.1$, rather than phase 0. Such a phase jitter is consistent with that observed so far from XB 1916-053: the 101 dip centers recorded by Chou et al. (2001) follow a distribution with a mean phase of 0.00098 and a $1 \sigma$ fluctuation of 0.06 . The dipping activity is associated with spectral hardening. This behavior is usual for XB 1916-053 and for most of the dippers. The dip shape varies from dip to dip. The dipping intensity shows erratic variability, ranging between $\sim 7 \%$ and $\sim 85 \%$ of the persistent level. Figure 2 shows an expanded view of the fourth complete dip observed with a time resolution of $4 \mathrm{~s}$. At times, the dipping is almost total in the $0.6-10 \mathrm{keV}$ energy range when plotted with this time resolution. Secondary dipping activity occuring at phase $\sim 0.6$ (i.e. $\sim 0.5$ away from the dip centers) is also visible, especially at $\sim 5.65 \mathrm{~h}$. The $0.6-10 \mathrm{keV}$ persistent intensity (outside the dips) decreased slowly throughout the observation from $\sim 67$ to $\sim 62$ counts s $^{-1}$.

\subsection{EPIC pn spectra}

\subsubsection{Persistent emission}

Intervals flagged with thick horizontal lines in Fig. 1 were selected as the persistent emission for the EPIC pn spectral 


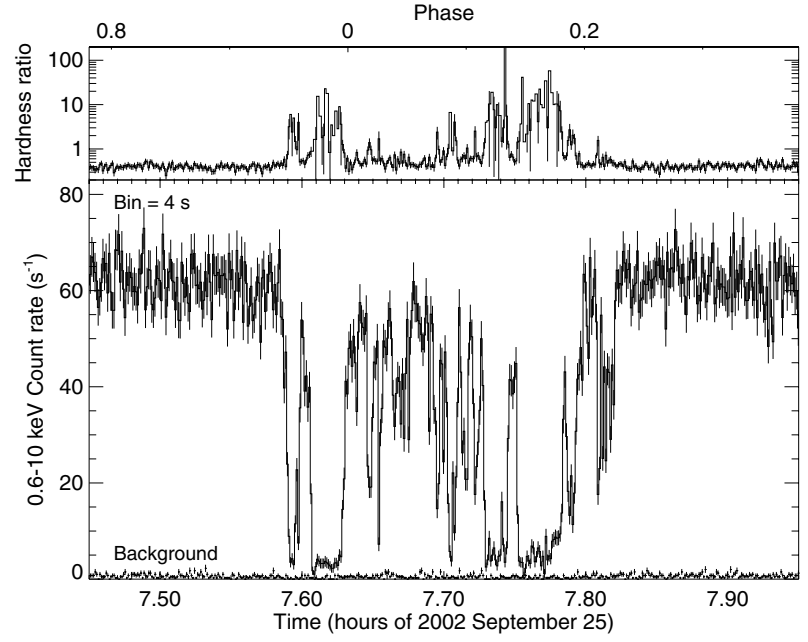

Fig. 2. A $0.6-10 \mathrm{keV}$ pn background-subtracted lightcurve of the dip seen on September 25, 2002, at $\sim 7.7 \mathrm{~h}$ plotted with a time resolution of $4 \mathrm{~s}$, showing the rapid intensity variability. Times are barycentercorrected. The $0.6-10 \mathrm{keV}$ background light curve taken from region adjacent to the source is also shown. The upper panel shows the hardness ratio (counts between $2.5-10 \mathrm{keV}$ divided by those between $0.6-$ $2.5 \mathrm{keV}$ ). The phase according to the Chou et al. (2001) ephemeris is indicated.

analysis. They correspond to phases in the range $0.25-0.92$. The interval corresponding to the intermediate dipping activity visible near $5.7 \mathrm{~h}$ has been excluded. The resulting persistent emission pn spectrum has an exposure of $9.9 \mathrm{ks}$.

An absorbed disk-blackbody plus power-law model fits the overall continuum reasonably well. The reduced $\chi^{2}\left(\chi_{v}^{2}\right)$ is 1.48 for 229 degrees of freedom (d.o.f.). The absorption, $N_{\mathrm{H}}$, is $(0.53 \pm 0.01) \times 10^{22}$ atom $\mathrm{cm}^{-2}$, the blackbody effective temperature at the innermost radius, $k T_{\text {in }}$, is $3.2 \mathrm{keV}$ and $\alpha$ is 2.9 . We note that the disk-blackbody component dominates the spectrum above $\sim 3 \mathrm{keV}$. It is probably over-estimated because of the lack of data above $10 \mathrm{keV}$. The inferred inner radius of the disk is remarkably small $(0.5 \mathrm{~km}$ assuming an inclination of $70^{\circ}$ ) and thus without any physical meaning. We caution that the power-law and disk-blackbody continuum model used here is not unique and other combinations of simple models can also provide acceptable fits. However, we focus here on the discovery of absorption features whose properties depend only weakly on the chosen continuum model. Detailed modelling of the continuum emission and discussion of its physical interpretation are the subject of a separate paper (Webb et al., in preparation). The power-law and disk-blackbody model allows the underlying continuum to be reliably modelled so that the absorption feature properties can be derived. Furthermore, this model allows the spectral changes between the persistent and the dipping emission to be modelled in a coherent way, within the framework of the complex continuum approach (see Sect. 3.2.2).

The observed count spectrum fit with the absorbed diskblackbody (3.2 keV) and power-law model ( $\alpha$ of 2.9) is shown in Fig. 3a. Examination of the residuals from this fit (Fig. 3b) reveals a structure around $0.9 \mathrm{keV}$ and two deep negative structures around $7 \mathrm{keV}$. The smaller structures at $\sim 1.8 \mathrm{keV}$ and $\sim 2.2 \mathrm{keV}$ are probably due to an incorrect instrumental modelling of the Si CCD and Au mirror edges.

In order to model the structure around $0.9 \mathrm{keV}$, a Gaussian emission feature with an energy of $0.87_{-0.03}^{+0.02} \mathrm{keV}$ and width $(\sigma)$ of $<74 \mathrm{eV}$ was first added to the previous model. This improves the fit ( $\chi_{v}^{2}$ of 1.35 for 227 d.o.f.). However, an even better fit is obtained if the Gaussian is replaced by an absorption edge at $0.98 \pm 0.02 \mathrm{keV}$ with an optical depth, $\tau$, of $0.10 \pm 0.02$. This results in a $\chi_{v}^{2}$ of 1.30 for 226 d.o.f. An F-test indicates that the probability for such an improvement occuring by chance is $1.7 \times 10^{-7}$. Thus, including an edge significantly improves the fit. We note that the RGS data support the edge interpretation independently using a different continuum model (see Sect. 3.3). The feature could be a $\mathrm{K}$ absorption edge from moderately ionized $\mathrm{Ne}$ ions, or an $\mathrm{L}$ absorption edge from moderately ionized $\mathrm{Fe}$ ions, or an edge complex from both kind of ions, since these astrophysically abundant elements have a series of photo-ionization thresholds around $0.9 \mathrm{keV}$ (the photoionization thresholds of $\mathrm{Ne} \mathrm{V} \mathrm{K}$ and of Fe X L being 0.987 and $0.959 \mathrm{keV}$, respectively).

The two deep negative residuals near $7 \mathrm{keV}$ were modelled using two narrow Gaussian absorption lines centered on $6.65 \mathrm{keV}$ and $6.95 \mathrm{keV}$. This results in a $\chi_{v}^{2}$ of 0.99 for 221 d.o.f. An F-test indicates that the probability for such an improvement occuring by chance is $2.0 \times 10^{-12}$. The measured energy of $6.65_{-0.02}^{+0.05} \mathrm{keV}$ of the first feature is consistent with that of $\mathrm{Fe} \mathrm{XxV} \mathrm{K} \alpha$, while the measured energy of $6.95_{-0.04}^{+0.05} \mathrm{keV}$ of the second feature is consistent with that of Fe XXVI K $\alpha$ (see Kotani et al. 2000, Table 5, for a list of absorption line candidates and references). Thus, we identify the observed absorption features with these resonant transitions from highly ionized Fe ions (He-like and H-like ions, respectively).

Table 1 gives the best-fit parameters of the model consisting of an edge $(0.98 \mathrm{keV})$, a disk-blackbody $(3.2 \mathrm{keV})$, a powerlaw ( $\alpha$ of 3.2) and two narrow absorption Gaussian lines (6.65 and $6.95 \mathrm{keV}$ ). This model and its components are shown together with the deconvolved spectrum in Fig. 3d. The residuals from this fit are shown in Fig. 3c. An expanded view of the residuals in the 4-10 keV range is shown in Fig. 4.

In addition to the clearly detected absorption features at $6.65 \mathrm{keV}$ and $6.95 \mathrm{keV}$ (Fig. 4, filled arrows), there is evidence for fainter absorption features at $2.67 \mathrm{keV}, 7.82 \mathrm{keV}$ and $8.29 \mathrm{keV}$ (Figs. 3c and 4). When absorption Gaussian lines are included, their derived energies are $2.67_{-0.07}^{+0.04} \mathrm{keV}$, $7.82_{-0.07}^{+0.03} \mathrm{keV}$ and $8.29_{-0.12}^{+0.08} \mathrm{keV}$, and their derived $E W \mathrm{~s}$ are $-6 \pm 3 \mathrm{eV},-21_{-12}^{+9} \mathrm{eV}$ and $-19_{-12}^{+11} \mathrm{eV}$, respectively. Although these features are marginal, it is interesting to note that the measured energies are consistent with that of S XVI, Ni XXVII $\mathrm{K} \alpha$ and Fe XXVI K $\beta$, respectively. Ni XXVII K $\alpha$ and Fe XXVI K $\beta$ features have been seen in the ASCA spectrum of GRS 1915+105 (Kotani et al. 2000), and there was evidence for their presence in the XMM-Newton spectrum of X 1624-490 (Parmar et al. 2002) too. A P Cygni S XVI feature is reported in Cir X-1 (Brandt \& Schulz 2000). Thus, we consider the marginal detections of absorption features at 2.67, 7.82 and $8.29 \mathrm{keV}$ in the spectrum of XB 1916-053 as evidence for the possible presence of S XVI, Ni XXVII K $\alpha$ and Fe XXVI $\mathrm{K} \beta$ absorption lines. 

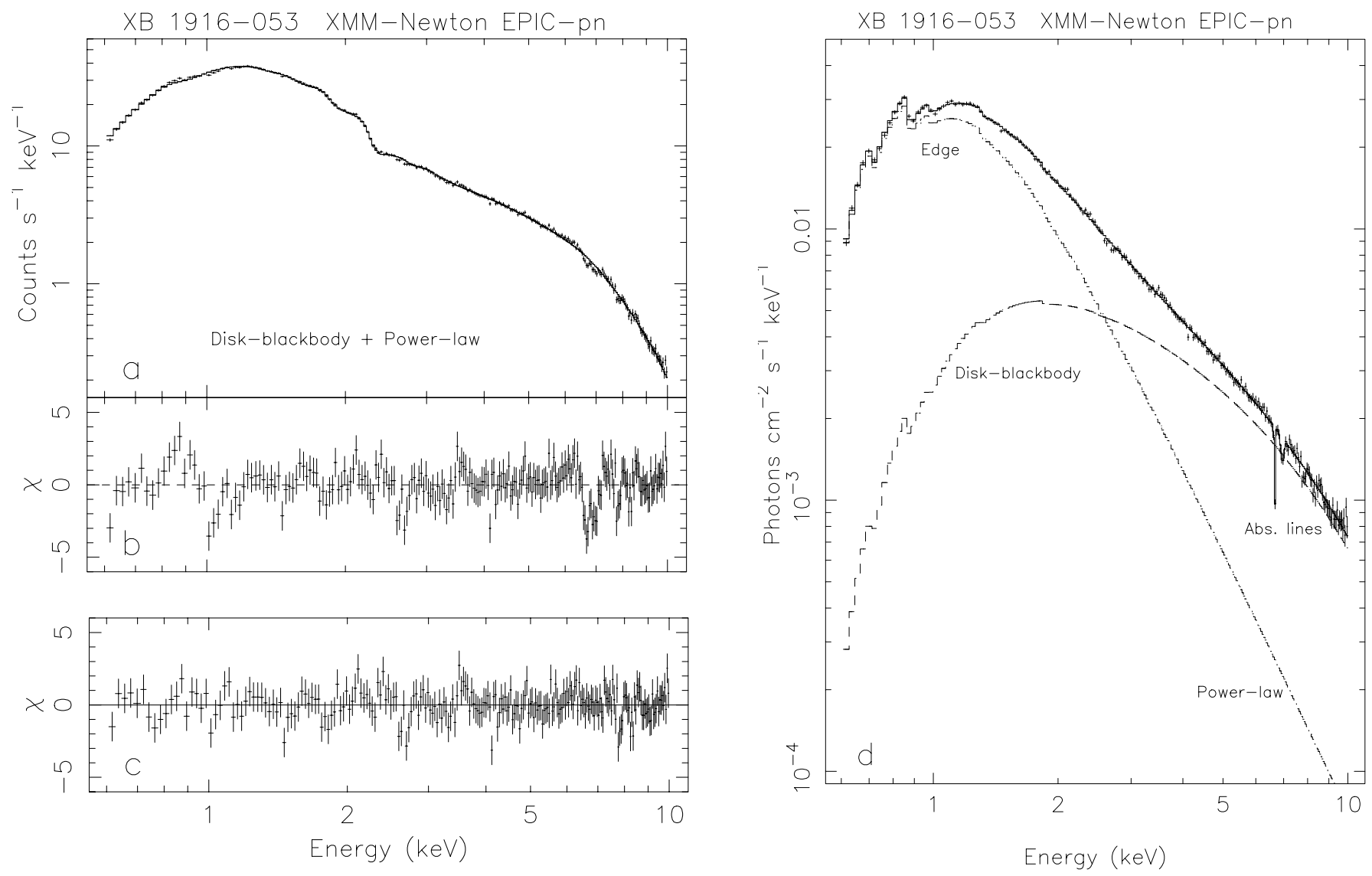

Fig. 3. a) pn spectra of the XB 1916-053 persistent emission and the best-fit disk-blackbody plus power-law continuum model; b) best-fit residuals which reveal the presence of a broad feature centered near $0.9 \mathrm{keV}$, together with narrow absorption features at 6.65 and $6.95 \mathrm{keV}$; c) residuals when an edge at $0.98 \mathrm{keV}$ and two absorption Gaussian lines at 6.65 and $6.95 \mathrm{keV}$ are included in the spectral model; d) deconvolved photon spectrum and the best-fit model including an edge at $0.98 \mathrm{keV}$ and two absorption lines at 6.65 and $6.95 \mathrm{keV}$. The individual components are indicated. The best-fit parameters for this model are given in Table 1. The other absorption edges visible in the spectrum are attributed to neutral material in the interstellar medium.

We have searched for $\mathrm{K}$ absorption edges in the pn persistent emission spectrum due to neutral Fe, Fe XXV and Fe XXVI expected at $7.12,8.76$ and $9.28 \mathrm{keV}$. The upper-limits to the optical depth of an edge at $7.12 \mathrm{keV}, 8.76 \mathrm{keV}$ and $9.28 \mathrm{keV}$ are $0.05,0.06$ and 0.02 , respectively.

As a broad emission feature at $\sim 6 \mathrm{keV}$ interpreted as fluorescent line emission from neutral $\mathrm{Fe}$ was detected in several spectra from XB 1916-053, we tried to include one in the model. Including a Gaussian emission feature with an energy of $6.6_{-0.6}^{+0.2} \mathrm{keV}$ and width $450 \pm 350 \mathrm{eV}$ to the model described in Table 1 results in a $\chi_{v}^{2}$ of 0.96 for 218 d.o.f. An F-test indicates that the probability for such an improvement occuring by chance is 0.035 . Thus, the fit is only marginally improved when the emission line is included. Therefore, the emission line was not kept in the model. We note however that the significance of such an emission line depends on the model chosen for the underlying continuum. If the high energy part of the spectrum is modelled using a cut-off power-law instead of a disk-blackbody as in the previous model, then the inclusion of a Gaussian emission line at $\sim 6 \mathrm{keV}$ significantly improves the fit. For instance, a good fit ( $\chi_{v}^{2}$ of 0.99 for 217 d.o.f.) of the spectrum is obtained using a model consisting of a disk-blackbody with $k T_{\text {in }}$ of $\sim 0.3 \mathrm{keV}$, a cut-off power-law with $\alpha$ of $\sim 1.6$ and $E_{\mathrm{c}}$ of $\sim 15 \mathrm{keV}$, in addition to an edge near $0.98 \mathrm{keV}$ and two absorption lines near 6.65 and $6.95 \mathrm{keV}$ with similar properties as in Table 1, and in addition to a Gaussian emission line with an energy of $6.2 \pm 0.3 \mathrm{keV}$ and width of $980_{-280}^{+450} \mathrm{eV}$. An F-test indicates that the probability of improving the fit by chance by adding the latter line is $3 \times 10^{-7}$. So, the Gaussian emission line is highly significant in that case. This difference illustrates rather well the difficulties of spectral fitting with moderate spectral resolution CCD data. The presence of the emission line is further discussed in the separate paper devoted to the continuum modelling.

\subsubsection{Dipping emission}

Intervals with phases in the range $0.92-0.25$ were used to investigate the dipping emission. Within these dipping intervals, three spectra were extracted based on intensity selection criteria. Events corresponding to a background-subtracted pn 0.6$10 \mathrm{keV}$ count rate in the range 40-60, 20-40 and 0-20 s $\mathrm{s}^{-1}$ (see Fig. 1) were extracted to form "shallow", "intermediate", and "deep" dipping spectra, respectively.

To quantify the spectral evolution from persistent to deep dipping emission and extract the properties of lines during 
Table 1. Best-fit to the $0.6-10 \mathrm{keV}$ XB $1916-053$ pn persistent emission spectrum using a disk-blackbody model with an inner temperature $k T_{\text {in }}$ and normalization $k_{\mathrm{DBB}}$ given by $\left(R_{\mathrm{in}} / d\right)^{2} \cos i$ where $R_{\text {in }}$ in the inner radius in $\mathrm{km}, d$ the distance in units of $10 \mathrm{kpc}$ and $i$ the inclination and a power-law with a photon index, $\alpha$ and a normalization at $1 \mathrm{keV}, k_{\mathrm{PL}}$, in unit of $\mathrm{ph} . \mathrm{keV}^{-1} \mathrm{~cm}^{-2} \mathrm{~s}^{-1}$, together with an edge with an energy, $E_{\text {edge }}$, and an optical depth, $\tau$, and two Gaussian absorption lines with their energy, $E_{\text {line }}$, width, $\sigma$ and equivalent width, $E W$.

\begin{tabular}{|c|c|c|}
\hline \multicolumn{3}{|c|}{ EPIC pn persistent emission } \\
\hline Component & Parameter & Value \\
\hline \multirow[t]{2}{*}{ Disk-blackbody } & $k T_{\text {in }}(\mathrm{keV})$ & $3.18 \pm 0.08$ \\
\hline & $k_{\mathrm{DBB}}$ & $0.106_{-0.006}^{+0.012}$ \\
\hline \multirow[t]{2}{*}{ Power-law } & $\alpha$ & $3.20 \pm 0.09$ \\
\hline & $k_{\mathrm{PL}}$ & $0.113 \pm 0.006$ \\
\hline \multirow[t]{2}{*}{ Edge } & $E_{\text {edge }}(\mathrm{keV})$ & $0.98 \pm 0.02$ \\
\hline & $\tau$ & $0.11 \pm 0.03$ \\
\hline $\mathrm{Fe} \mathrm{XXV} \mathrm{K} \alpha$ & $E_{\text {line }}(\mathrm{keV})$ & $6.65_{-0.02}^{+0.05}$ \\
\hline absorption & $\sigma(\mathrm{eV})$ & $<100$ \\
\hline Gaussian & $E W(\mathrm{eV})$ & $-30_{-12}^{+8}$ \\
\hline Fe XXVI K $\alpha$ & $E_{\text {line }}(\mathrm{keV})$ & $6.95_{-0.04}^{+0.05}$ \\
\hline absorption & $\sigma(\mathrm{eV})$ & $<140$ \\
\hline Gaussian & $E W(\mathrm{eV})$ & $-30_{-12}^{+11}$ \\
\hline \multicolumn{2}{|c|}{$N_{\mathrm{H}}\left(\right.$ atom $\left.\mathrm{cm}^{-2}\right)$} & $(0.59 \pm 0.02) \times 10^{22}$ \\
\hline \multicolumn{2}{|c|}{$\chi_{v}^{2}$ (d.o.f.) } & $0.99(221)$ \\
\hline \multicolumn{2}{|c|}{$L_{0.6-10 \mathrm{keV}}\left(\mathrm{erg} \mathrm{s}^{-1}\right.$, at $\left.9.3 \mathrm{kpc}\right)$} & $4.5 \times 10^{36}$ \\
\hline
\end{tabular}

dipping, the complex continuum approach was used. Discussion on other possible continuum models is reserved to a separate paper. Our reference model for the complex continuum approach is the best-fit to the persistent spectrum using a model including the edge, the disk-blackbody and the power-law components. The best-fit $N_{\mathrm{H}}$ (applied to both components $)$ is $(0.58 \pm 0.02) \times 10^{22}$ atom $\mathrm{cm}^{-2}, k T_{\text {in }}$ is $3.13_{-0.08}^{+0.09} \mathrm{keV}, \alpha$ is $3.17 \pm 0.09, E_{\text {edge }}$ is $0.98 \pm 0.02 \mathrm{keV}$ and $\tau$ is $0.10 \pm 0.03$ (see also Table 3). We applied the complex continuum model in the following form: $\mathrm{Edge} *\left[\mathrm{AB}^{\mathrm{DBB} *} \mathrm{DBB}+\mathrm{AB}^{\mathrm{Gal} *}\left[\mathrm{AB}^{\mathrm{PL} *} f+(1-f)\right] * \mathrm{PL}\right]$, where DBB represents the disk-blackbody component (pointsource), and PL the power-law component (extended). $f$ is the covering fraction $(0 \leqq f \leqq 1)$. The $\mathrm{AB}$ terms represent absorption of the form $\mathrm{e}^{-\sigma N_{\mathrm{H}}}$ where $\sigma$ are the photoelectric cross-sections and $N_{\mathrm{H}}$ the column density. $\mathrm{AB}^{\mathrm{Gal}}$ represents Galactic absorption. $\mathrm{AB}^{\mathrm{PL}}$ reprensents the absorption applied to the covered fraction of the power-law emission, in addition to the Galactic absorption. $\mathrm{AB}^{\mathrm{DBB}}$ represents the absorption applied to the disk-blackbody component and includes Galactic absorption.

$N_{\mathrm{H}}^{\mathrm{Gal}}$ was fixed to $0.58 \times 10^{22}$ atom $\mathrm{cm}^{-2}$, the value obtained from the fit to the persistent spectrum. When $f$ is

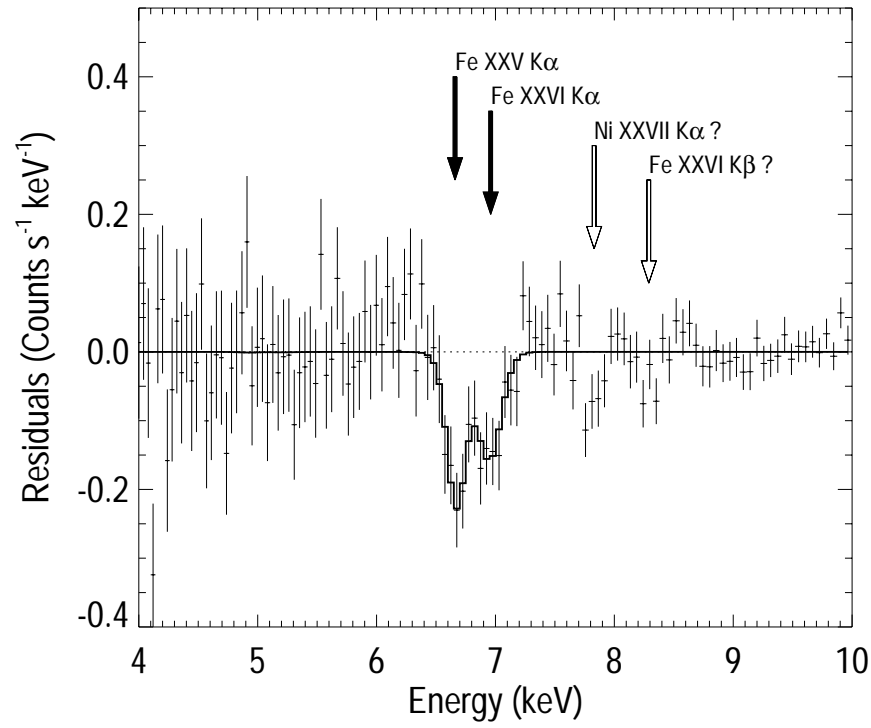

Fig. 4. Residuals in the 4-10 keV energy range when the best-fit model given in Table 1 is fit to the pn spectrum of the XB 1916-053 persistent emission. The normalizations of the narrow absorption features at $6.65 \mathrm{keV}$ and $6.95 \mathrm{keV}$ have been set to zero. These two absorption features, identified with Fe XXV K $\alpha$ and Fe XXVI $\mathrm{K} \alpha$, are shown with filled arrows. Fainter absorption features shown with non-filled arrows at 7.83 and $8.29 \mathrm{keV}$ may be present and have energies consistent with Ni XXVII K $\alpha$ and Fe XXVI K $\beta$ transitions.

set to 0, i.e. when the power-law component is uncovered, the complex continuum model is equivalent to our reference model applied to the persistent emission, as expected. To fit each intensity-selected dipping spectrum, only $f, N_{\mathrm{H}}^{\mathrm{DBB}}$, and $N_{\mathrm{H}}^{\mathrm{PL}}$ are allowed to vary, whereas the parameters and normalizations of the disk-blackbody and power-law components are fixed to their persistent emission values. The parameters of the edge (energy and optical depth) were also fixed to the persistent emission values. This gave reasonably good fit to each dipping spectrum, with $\chi_{v}^{2}$ values of 1.28 (227 d.o.f.), 2.03 (225 d.o.f.) and 1.30 (217 d.o.f.) for the shallow, intermediate and deep dipping spectra, respectively. However, inspection of the residuals from this fits reveals broad structures below $\sim 1 \mathrm{keV}$. These structures are well accounted for if the edge parameters are allowed to vary, instead of being fixed to the persistent emission values. When the edge parameters are allowed to vary, the $\chi_{v}^{2}$ values become 1.14 (225 d.o.f.), 1.57 (223 d.o.f.) and 1.26 (215 d.o.f.) for the shallow, intermediate and deep dipping spectra, respectively. F-tests indicate that the probability for such an improvement occuring by chance is $8.2 \times 10^{-7}, 1.1 \times 10^{-13}$ and $6.6 \times 10^{-2}$, respectively. Thus, while the fit of the deep dipping spectrum is only marginally improved, the fit of the shallow and intermediate spectra are significantly improved when the edge parameters are allowed to vary. The intensity-selected dipping spectra fit in such a way are shown in Fig. 5. The corresponding best-fit parameters are given in Table 3 and plotted as a function of intensity in Fig. 7. The persistent emission parameters are also shown for comparison. $f, N_{\mathrm{H}}^{\mathrm{PL}}$ and $N_{\mathrm{H}}^{\mathrm{DBB}}$ are all observed to increase as XB 1916-053 evolves from its persistent to its deep dipping state. The power-law emission is progressively covered until 


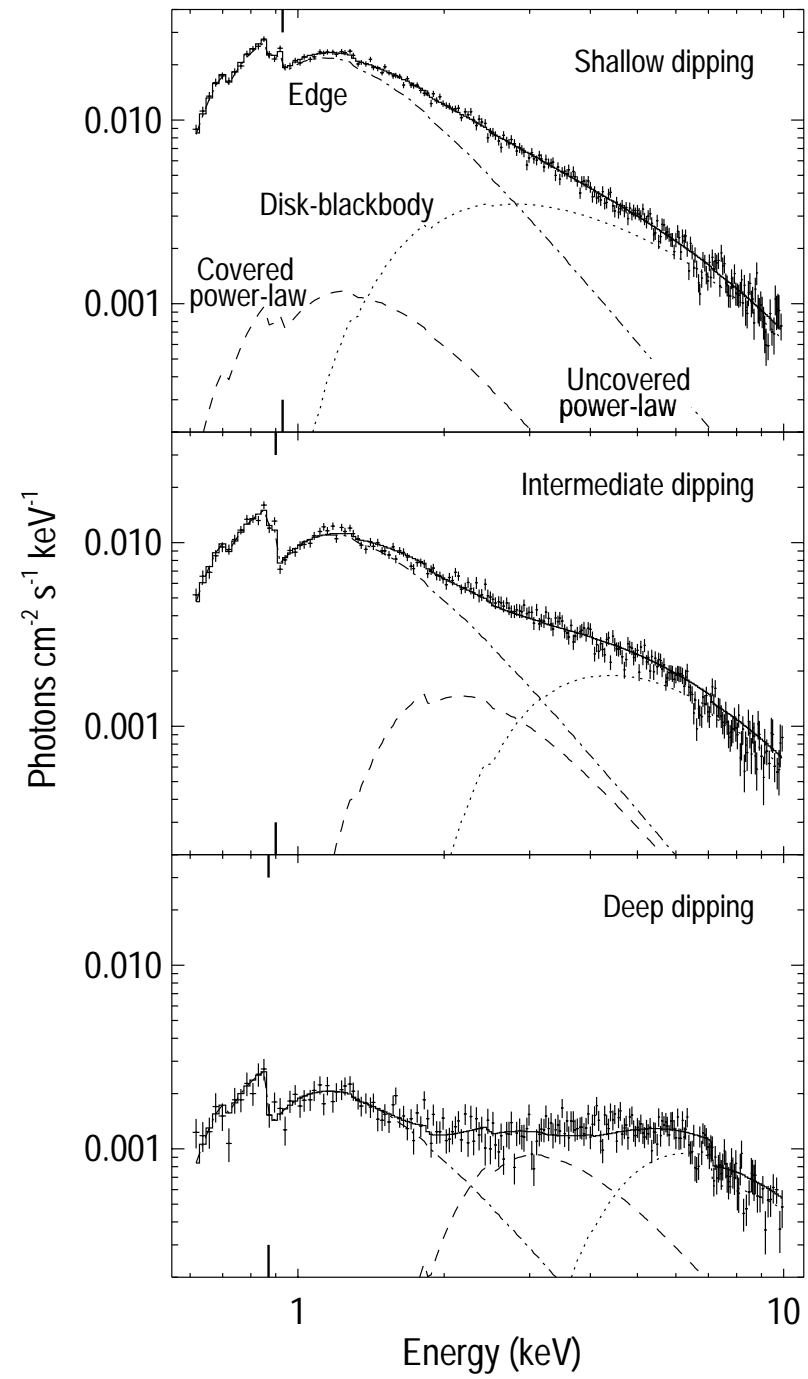

Fig. 5. Deconvolved pn intensity-selected dipping spectra of XB 1916-053 fit using a complex continuum model. The different components of the model are indicated in the upper panel: the edge, the disk-blackbody (dotted line), the power-law partly covered (dashed line) and partly uncovered (dashed-dotted line). Vertical tick marks indicate the position of the edge in each spectrum. The decrease of the edge energy as XB 1916-053 evolves from shallow to deep dipping is clearly visible.

Table 2. Spectral parameters of the Fe XXV K $\alpha$ absorption line detected during intensity-selected dipping intervals. The feature is modelled as a Gaussian line added to the disk-blackbody component of the complex continuum model. $E_{\text {line }}, \sigma$ and $E W$ are the line energy, width and equivalent width, respectively.

\begin{tabular}{cccc}
\hline \hline \multicolumn{4}{c}{ EPIC pn dipping emission } \\
\hline Shallow & Intermediate & Deep \\
\hline Fe XXV K $\alpha$ absorption Gaussian \\
$E_{\text {line }}(\mathrm{keV})$ & $6.70 \pm 0.05$ & $6.67_{-0.05}^{+0.06}$ & $6.59 \pm 0.05$ \\
$\sigma(\mathrm{eV})$ & $<110$ & $160_{-60}^{+80}$ & $<173$ \\
$E W(\mathrm{eV})$ & $-74_{-27}^{+20}$ & $-168_{-46}^{+44}$ & $-119_{-50}^{+45}$ \\
\hline
\end{tabular}

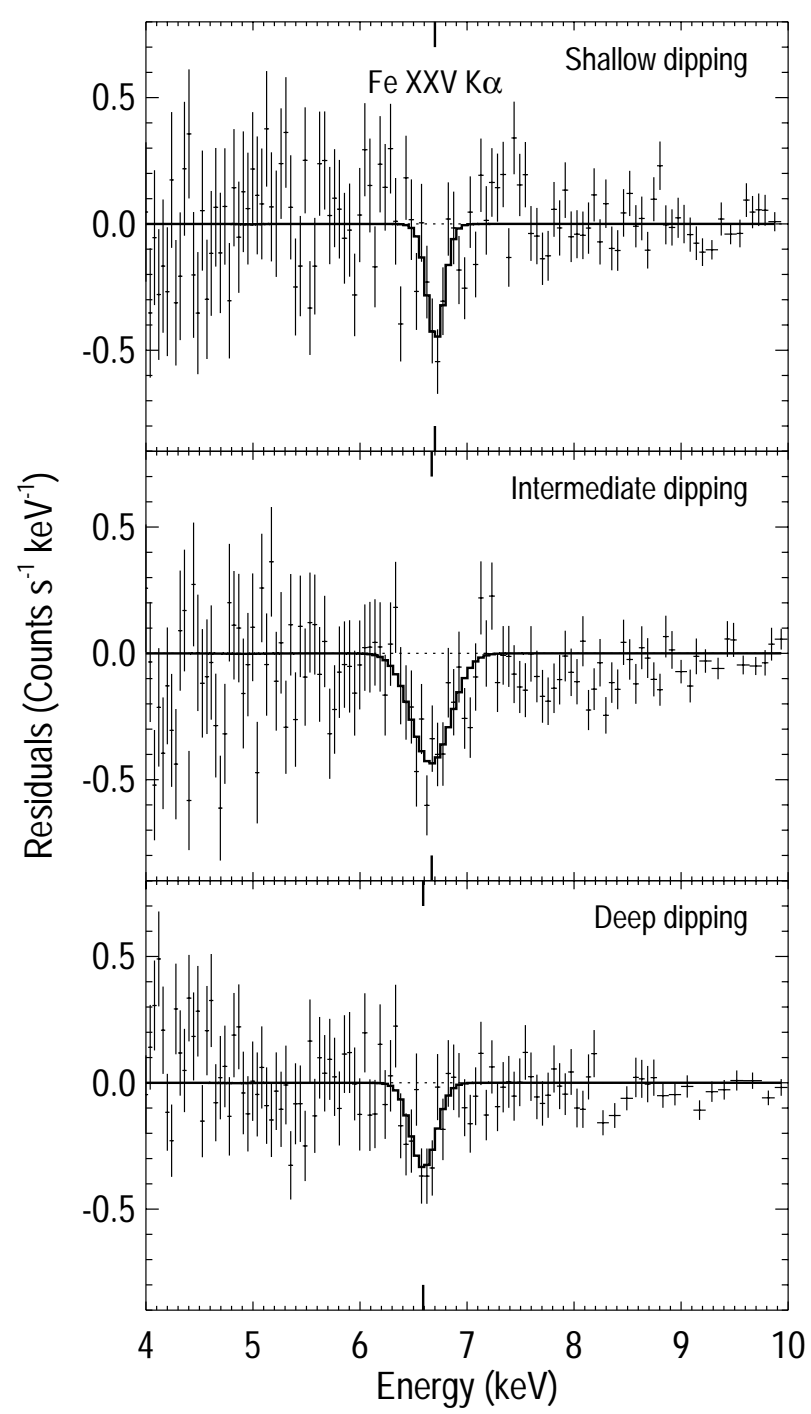

Fig. 6. $4-10 \mathrm{keV}$ residuals when the best-fit complex continuum model and absorption lines are fit to the pn intensity-selected XB 1916-053 dipping spectra. The normalizations of the narrow absorption features have been set to 0 . An absorption feature near $6.7 \mathrm{keV}$, identified with $\mathrm{Fe} \mathrm{XXV} \mathrm{K} \alpha$, is detected in each spectrum and its energy is indicated by vertical tick marks.

$f$ reaches $91 \%$ and $N_{\mathrm{H}}^{\mathrm{PL}} 7.5 \times 10^{22}$ atom $\mathrm{cm}^{-2}$ in the deepest dipping spectrum. The disk-blackbody emission is strongly absorbed, with $N_{\mathrm{H}}^{\mathrm{DBB}}$ reaching $34 \times 10^{22}$ atom $\mathrm{cm}^{-2}$ in the deepest dipping spectrum. Thus, dipping is well accounted for by large increases of column density for the point-like diskblackbody emission, combined with the extended power-law emission being progressively covered by the absorber. This is consistent with previous results of the complex continuum approach applied to XB 1916-053 and other dipping sources (e.g., Church et al. 1997). An interesting new result is the decrease of the edge energy as XB 1916-053 evolves from its persistent towards its deepest dipping state. Assuming the edge actually represents a complex of edges from ions in a range of ionization states, the decrease of energy is consistent with a decrease in the average ionization level. This suggests that during dipping, the additional absorbing material is less ionized than during the persistent emission. The increase of the edge optical 
Table 3. Best-fits to the intensity-selected dipping spectra using the complex continuum model (see text). The best-fit to the persistent spectrum used as a basis for the complex continuum approach is also given for comparison.

\begin{tabular}{lccccccc}
\hline \hline EPIC pn & $\begin{array}{c}N_{\mathrm{H}}^{\mathrm{DBB}} \\
\left(10^{22} \mathrm{~cm}^{-2}\right)\end{array}$ & $\begin{array}{c}N_{\mathrm{H}}^{\mathrm{PL}} \\
\left(10^{22} \mathrm{~cm}^{-2}\right)\end{array}$ & $f$ & $\begin{array}{c}E_{\text {edge }} \\
(\mathrm{keV})\end{array}$ & $\tau$ & $\chi_{v}^{2}$ & d.o.f. \\
\hline Persistent emission & \multicolumn{2}{c}{$N_{\mathrm{H}}=(0.58 \pm 0.02)$} & $(0)$ & $0.98 \pm 0.02$ & $0.10 \pm 0.03$ & 1.30 & 227 \\
Shallow dipping & $1.93_{-0.07}^{+0.12}$ & $0.2_{-0.1}^{+0.3}$ & $0.07_{-0.07}^{+0.04}$ & $0.93 \pm 0.02$ & $0.27_{-0.05}^{+0.04}$ & 1.14 & 225 \\
Intermediate dipping & $8.8 \pm 0.4$ & $2.6_{-0.2}^{+0.3}$ & $0.47 \pm 0.02$ & $0.90_{-0.01}^{+0.02}$ & $0.54_{-0.08}^{+0.07}$ & 1.57 & 223 \\
Deep dipping & $34 \pm 1$ & $7.5 \pm 0.3$ & $0.907 \pm 0.006$ & $0.87_{-0.04}^{+0.06}$ & $0.5 \pm 0.2$ & 1.26 & 215 \\
\hline
\end{tabular}

depth from the persistent to the deepest dipping state is consistent with more absorbing material being present in the line of sight during dipping.

Examination of the intensity-selected dipping spectra (Fig. 5) reveals narrow absorption features near $6.7 \mathrm{keV}$. Adding a Gaussian line in absorption at $\sim 6.7 \mathrm{keV}$ to the complex continuum model gives $\chi_{v}^{2}$ of 0.99 (222 d.o.f.), 1.27 (220 d.o.f.) and 1.12 (212 d.o.f.) for the shallow, intermediate and deep dipping spectra, respectively. F-tests indicate that the probability for such an improvement occuring by chance is $3.5 \times 10^{-7}, 9.2 \times 10^{-11}$ and $4.3 \times 10^{-6}$, respectively. The properties of the lines are given in Table 2, and an expanded view of the residuals to the intensity-selected dipping spectra is shown in Fig. 6. The measured energies of the absorption lines are all around $6.7 \mathrm{keV}$ and thus consistent with that of Fe XXV $\mathrm{K} \alpha$, already detected in the persistent spectrum. However, there is some evidence for a slight decrease of the Gaussian energy as XB 1916-053 evolves from shallow to deep dipping. Considering that the observed absorption feature, with limited statistics and resolution, may not be only due to one ion, but may contain unresolved contributions from other ions, this trend, although marginal, is consistent with the absorbing material becoming less ionized from shallow to deep dipping. The absorption feature detected during the deep dipping spectrum could be due to the combination of $\mathrm{K} \alpha$ absorption lines from Fe XXV, Fe XXIV and less ionized ions, with a proportion of Fe XXIV and less ionized ions larger than during the intermediate dipping.

In addition to the clearly detected $\mathrm{FeXXV} \mathrm{K} \alpha$ absorption line, there is evidence for an absorption feature around $8.3 \mathrm{keV}$ in the deep dipping spectrum (Fig. 6). When an absorption line is included, its measured energy is $8.35 \pm 0.05 \mathrm{keV}$. This energy is close to but not consistent with that of $\mathrm{FeXXV} \mathrm{K} \beta$. There is no obvious feature expected to be strong at that energy. If the feature is modelled as an edge rather than a Gaussian line, the best-fit edge energy is $8.0_{-0.4}^{+0.3} \mathrm{keV}$ and $\tau=0.3 \pm 0.1$. A likely explanation for this marginal feature is a combination of $\mathrm{K}$ absorption edges from moderately ionized Fe (Fe XII and higher) and neutral or slightly ionized $\mathrm{Ni}$, with possibly the contribution of absorption lines as well.

We set upper-limits of $-54 \mathrm{eV},-72 \mathrm{eV}$ and $-50 \mathrm{eV}$ on the $E W$ of a narrow Fe XXVI K $\alpha$ in shallow, intermediate and deep dipping spectra, respectively (by including a Gaussian with an energy fixed to $6.97 \mathrm{keV}$, the rest energy of the transition, and a width fixed to 0 ). The marginally significant lines seen in the persistent spectrum are not evident in the dipping spectra.

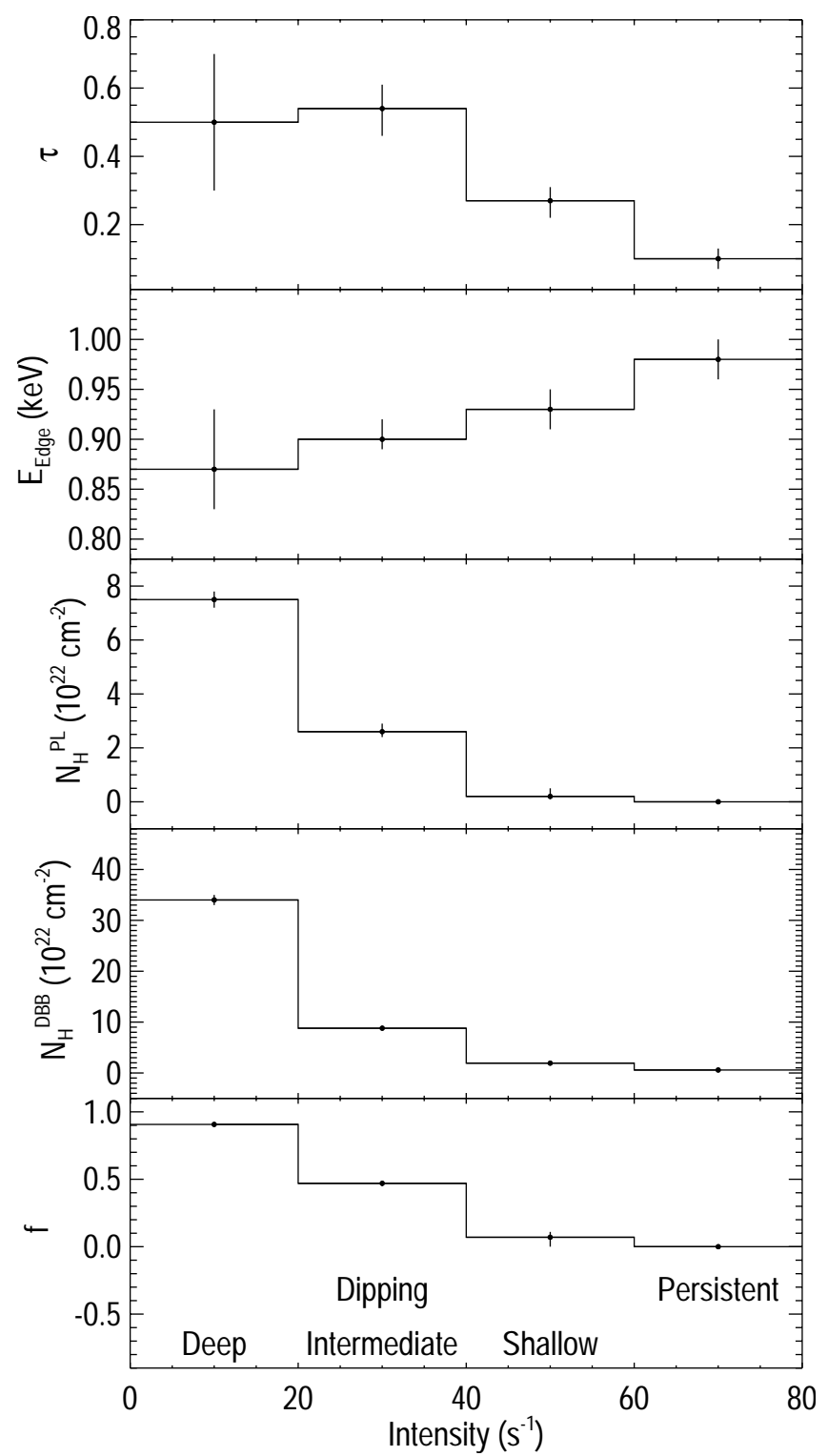

Fig. 7. Best-fit parameters to the intensity-selected dipping spectra using the complex continuum approach, plotted as a function of intensity (see text and Table 3). The best-fit parameters to the persistent spectrum using the basis model for the complex continuum approach are also shown.

\subsection{RGS spectra}

The dipping activity is clearly visible in the RGS light curve. The same intervals as used for the pn were used to extract persistent and dipping RGS spectra. We did not further divide the 
dipping intervals using intensity criteria as was done for pn, because of the low RGS count rate during the dips. RGS1 and RGS2 spectra were fit together with a unique model to better constrain spectral modelling, but the normalizations were allowed to vary independently to account for calibration differences. The ranges $0.903-1.177$ and $0.516-0.602 \mathrm{keV}$ are not present in the data from RGS1 and RGS2, respectively, because of malfunction of the drive electronics for one CCD chip in each spectrometer.

The persistent emission RGS spectrum was first modelled using an absorbed power-law. This results in a moderately good fit with a $\chi_{v}^{2}$ of 1.26 for 891 d.o.f. However, examination of the residuals reveals a structure around $0.9 \mathrm{keV}$ similar to the one observed in the pn spectrum and well accounted for by an edge (Fig. 3b and Table 1). Therefore, we included such an edge to the previous model of the RGS spectrum. This gives a $\chi_{v}^{2}$ of 1.22 for 889 d.o.f. An F-test indicates that the probability for such an improvement occuring by chance is $4.8 \times 10^{-7}$. Thus, the fit is significantly improved when the edge is included. The RGS persistent emission spectrum and the best-fit model using a power-law and an edge are shown in Fig. 8 (top panel), and the best-fit parameters are given in Table 4 . The edge energy of $0.99 \pm 0.02 \mathrm{keV}$ measured from the RGS spectrum is fully consistent with that obtained from the EPIC pn spectrum.

A good fit to the dipping emission RGS spectrum is obtained using an absorbed power-law model $\left(\chi_{v}^{2}\right.$ of 1.06 for 171 d.o.f.). As an edge around $0.9 \mathrm{keV}$ was detected in the dipping emission pn spectra, we included an edge to the previous model of the RGS spectrum. This gives a $\chi_{v}^{2}$ of 1.00 for 169 d.o.f. An F-test indicates that the probability for such an improvement occuring by chance is 0.0027 , indicating that the edge is detected at a $99.7 \%$ confidence level $(3 \sigma)$. The RGS dipping emission spectrum and the corresponding best-fit model using a power-law and an edge are shown in Fig. 8 (bottom panel), and the best-fit parameters are given in Table 4 . The measured edge energy of $0.90_{-0.03}^{+0.07} \mathrm{keV}$ obtained from the dipping RGS spectrum is consistent with the mean value obtained from the three intensity-selected dipping pn spectra (Table 3). Thus, RGS results strengthen the conclusion drawn from pn results that the edge energy is less during dipping emission than during persistent emission, which is consistent with the absorbing material being less ionized during dipping.

We note that the column densities obtained from the RGS persistent and dipping spectra $\left(N_{\mathrm{H}}\right.$ of $0.41 \times 10^{22}$ atom $\mathrm{cm}^{-2}$ and $0.51 \times 10^{22}$ atom $\mathrm{cm}^{-2}$, respectively) are roughly equal to each other and roughly consistent with that obtained from EPIC pn spectra. Indeed, the low-energy part of pn spectra (where RGS is sensitive) is dominated by the uncovered and unabsorbed component (here the power-law) of the complex continuum model (see Fig. 5). This component is only affected by the Galactic absorption, assumed to be $0.58 \times 10^{22}$ atom $\mathrm{cm}^{-2}$ within the complex continuum model. When $N_{\mathrm{H}}$ is forced to be $\geq 0.58 \times 10^{22}$ atom $\mathrm{cm}^{-2}$, almost as good fits as those presented in Table 4 are obtained to the RGS spectra, with best-fit $N_{\mathrm{H}}$ of $0.58 \times 10^{22}$ atom $\mathrm{cm}^{-2}$ in both cases, similar parameters for the edge and $\alpha$ of $\sim 2.7$ and $\sim 2.9$ in the persistent and dipping spectrum, respectively. No acceptable fit is found to

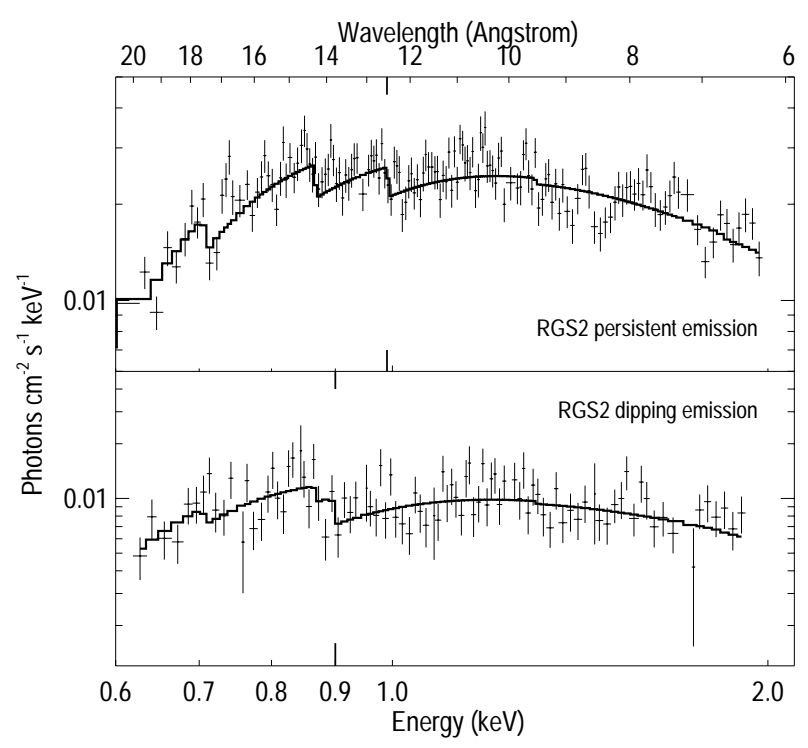

Fig. 8. Deconvolved RGS2 spectra of the XB 1916-053 persistent (top panel) and dipping emission (bottom panel) fit using a power-law and an edge model (thick line). The best-fit models were obtained by fitting RGS1 and RGS2 spectra simultaneously (see Table 4), but only RGS2 spectra are shown for clarity. Vertical tick marks indicate the position of the edge included in each spectrum.

Table 4. Best-fit to RGS persistent and dipping emission spectra of XB 1916-053 using a power-law model with a photon index, $\alpha$, together with an edge with an energy, $E_{\text {edge }}$, an optical depth, $\tau$. RGS1 and RGS2 spectra are fit together, but the RGS1 and RGS2 power-law normalizations at $1 \mathrm{keV}, k_{\mathrm{RGS} 1}$ and $k_{\mathrm{RGS} 2}\left(\mathrm{ph} . \mathrm{keV}^{-1} \mathrm{~cm}^{-2} \mathrm{~s}^{-1}\right.$ ), are allowed to vary independently.

\begin{tabular}{cccc}
\hline \hline RGS1 \& RGS2 & Persistent & Dipping \\
\hline Power-law & $\alpha$ & $2.4_{-0.1}^{+0.2}$ & $2.1_{-0.3}^{+0.4}$ \\
& $k_{\mathrm{RGS} 1}$ & $0.101_{-0.009}^{+0.01}$ & $0.036_{-0.007}^{+0.01}$ \\
& $k_{\mathrm{RGS} 2}$ & $0.092_{-0.009}^{+0.008}$ & $0.031_{-0.006}^{+0.008}$ \\
\multirow{2}{*}{ Edge } & $E_{\text {edge }}(\mathrm{keV})$ & $0.99 \pm 0.02$ & $0.90_{-0.03}^{+0.07}$ \\
& $\tau$ & $0.22 \pm 0.06$ & $0.3_{-0.1}^{+0.2}$ \\
\multicolumn{2}{c}{$N_{\mathrm{H}}\left(10^{22} \mathrm{~cm}^{-2}\right)$} & $0.51 \pm 0.03$ & $0.42 \pm 0.07$ \\
\multicolumn{2}{c}{$\chi_{v}^{2}$ (d.o.f.) } & $1.22(889)$ & $1.00(169)$ \\
\hline
\end{tabular}

the RGS dipping spectrum by fixing $N_{\mathrm{H}}$ to an higher value (e.g. $2 \times 10^{22}$ atom $\mathrm{cm}^{-2}$ ), since such an absorption implies basically no counts below $1 \mathrm{keV}$, whereas counts below $1 \mathrm{keV}$ are actually present.

An absorption feature is evident near $1.5 \mathrm{keV}(8.5 \AA)$ in the RGS2 persistent emission spectrum shown in Fig. 8. However this feature is less obvious in RGS1. The inclusion of a Gaussian absorption line with an energy of $1.48 \pm 0.01 \mathrm{keV}$, a width of $<41 \mathrm{eV}$, and an $E W$ of $-7 \pm 3 \mathrm{eV}$ in the combined RGS spectrum is marginally significant. However, it is interesting to note that the measured energy is consistent with that of $\mathrm{Mg}$ XII $\mathrm{K} \alpha$ at $1.47 \mathrm{keV}$. Thus, we consider that there is marginal evidence for the presence of an absorption line from 
Table 5. X-ray binaries known to exhibit X-ray absorption lines from highly ionized ( $\mathrm{H}$ - and $\mathrm{He}-\mathrm{like}) \mathrm{Fe}$ and other metals, ordered by increasing orbital period. The source name is given in Col. 1. The orbital period taken from Ritter \& Kolb (2003, see references therein) catalog, unless otherwhise mentioned, is given in Col. 2. Column 3 indicates if dips (D) and eclipses (E) are observed from the source. The system inclination, $i$, is given in Col. 4. It is deduced from the presence of dips or eclipses after Frank et al. (1987), unless otherwhise mentioned. The H-like and/or He-like ions detected in each source are listed in Col. 5, and the corresponding observatory and references used are given in Cols. 6 and 7.

\begin{tabular}{lllllll}
\hline \hline Source & $P_{\text {orb }}(\mathrm{h})$ & Dips & $i\left(^{\circ}\right)$ & H- or He-like ions & Observatory & References \\
\hline XB 1916-053 & 0.8 & $\mathrm{D}$ & $60-80$ & $\mathrm{Fe}$ & XMM & This work \\
X 1254-690 & 3.9 & $\mathrm{D}$ & $60-80$ & $\mathrm{Fe}$ & XMM & Boirin \& Parmar (2003) \\
MXB 1658-298 & 7.1 & $\mathrm{D}, \mathrm{E}$ & $\sim 80$ & $\mathrm{O}, \mathrm{Ne}, \mathrm{Fe}$ & XMM & Sidoli et al. (2003) \\
X 1624-490 & 20.9 & $\mathrm{D}$ & $60-80$ & $\mathrm{Fe}$ & XMM & Parmar et al. (2002) \\
GRO J1655-401 & $62.9^{2}$ & $\mathrm{D}^{3}$ & $69.5 \pm 0.3^{2}$ & $\mathrm{Fe}$ & ASCA & Ueda et al. (1998); Yamaoka et al. (2001) \\
Cir X-1 & $398^{4}$ & $\mathrm{D}^{5}$ & high $?^{6}$ & $\mathrm{Ne}, \mathrm{Mg}, \mathrm{Si}, \mathrm{S}, \mathrm{Fe}$ & Chandra & Brandt \& Schulz (2000) \\
GX 13+1 & $602^{8}$ & no & low $?^{9}$ & $\mathrm{Ca}, \mathrm{Fe}, \mathrm{Ni}$ & ASCA, XMM & Ueda et al. (2002); Sidoli et al. (2002) \\
GRS 1915+105 ${ }^{1}$ & 804 & no & $\sim 70^{10}$ & $\mathrm{Ca}, \mathrm{Fe}, \mathrm{Ni}$ & ASCA, Chandra & Kotani et al. (2000); Lee et al. (2002) \\
\hline
\end{tabular}

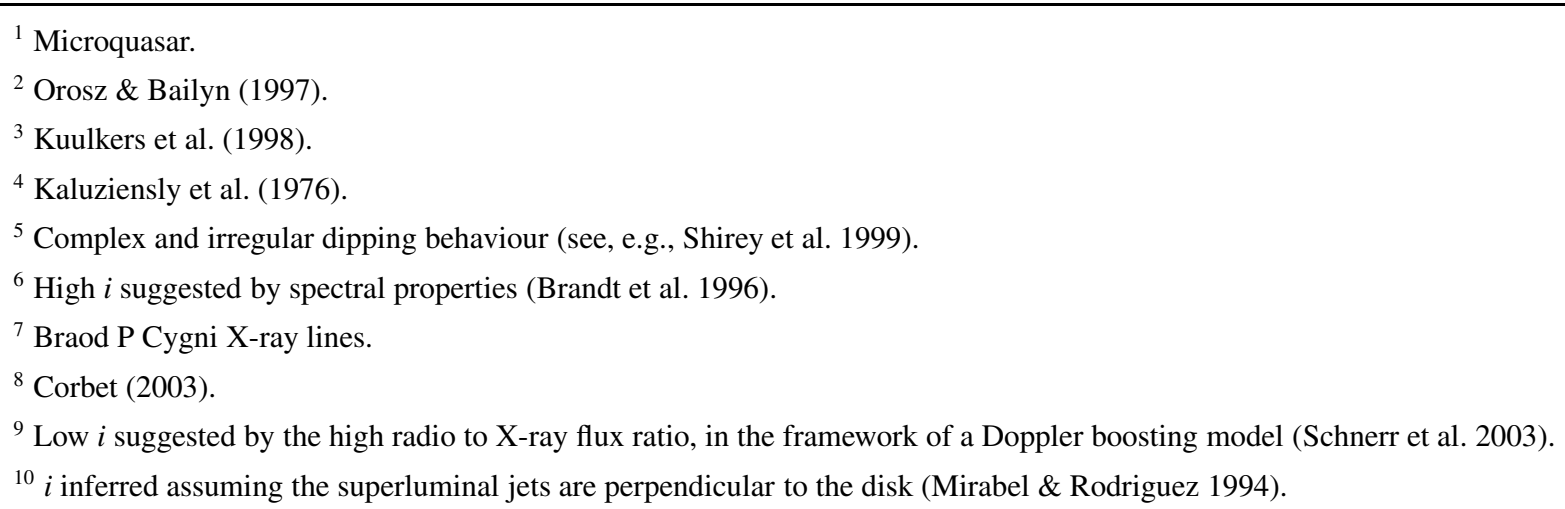

highly ionized Mg (H-like) in XB 1916-053. The upper-limit on the $E W$ of a narrow $\mathrm{Mg}$ XII $\mathrm{K} \alpha$ absorption line in the pn persistent spectrum is $-4.5 \mathrm{eV}$. The upper-limit on the $E W$ of a narrow $\mathrm{Mg}$ XII $\mathrm{K} \alpha$ absorption line in the RGS dipping spectrum is $-18 \mathrm{eV}$. The upper-limits on a narrow $\mathrm{Ne} \mathrm{X}$ absorption line at $1.02 \mathrm{keV}(12.19 \AA)$, a feature detected e.g. in MXB 1658-298 (Sidoli et al. 2001), are -5 and $-9 \mathrm{eV}$ in the RGS persistent and dipping spectra, respectively.

\section{Discussion}

We have modelled the EPIC pn continuum of XB 1916-053 during persistent emission intervals using a combination of disk-blackbody and power-law models together with an absorption edge at $0.98 \mathrm{keV}$ and narrow absorption lines at 6.65 and $6.95 \mathrm{keV}$. We have characterized the spectral changes in the EPIC pn data during dipping intervals following the complex continuum approach, and using the same continuum components in order to investigate the properties of the narrow absorption features. Dipping is well accounted for by large increases in $N_{\mathrm{H}}$ of the point-like disk-blackbody emission, combined with the extended power-law emission being progressively covered by the absorber. The edge energy decreases from $0.98 \mathrm{keV}$ to $0.87 \mathrm{keV}$ as $\mathrm{XB} 1916-053$ evolves from persistent to deep dipping. This result is consistent with that obtained from RGS data. This suggests that the detected edge represents a complex of edges from moderately ionized $\mathrm{Ne}$ and/or $\mathrm{Fe}$, and that the average ionization level decreases from persistent to deep dipping. This implies that during dipping, the absorbing material in the line of sight is less ionized than during the persistent emission. Photo-ionization is likely to be the dominant ionization process in X-ray binaries because of the high radiation field. Since the photo-ionization parameter, $\xi=L / n_{\mathrm{e}} r^{2}$ (e.g., Kallman \& McCray 1982), where $L$ is the luminosity of the ionizing source, $n_{\mathrm{e}}$ the electron density and $r$ the distance to the ionizing source, depends on $r^{-2}$ and $n_{\mathrm{e}}{ }^{-1}$, the decrease of the average ionization level during dipping indicates that the additional absorbing material during dipping is either more distant from the ionizing source and/or denser than the absorbing material present during persistent emission intervals. This supports the model where the dips are due to relatively cold clouds near the outer edge or circularization radius, of the disk.

Table 5 lists the X-ray binaries where X-ray absorption lines from highly ionized $\mathrm{Fe}$ or other metals have been reported. We note that Cir X-1 differs from the other sources by showing broad X-ray lines with P Cygni profiles. The eclipsing, dipping and bursting LMXB EXO 0748-676, has shown X-ray absorption lines from $\mathrm{H}$ - and He-like ions, but these lines differ from those found in the other sources in being redshifted, detected during X-ray bursts and attributed to the neutron star photosphere (Cottam et al. 2002). Therefore, we did not include EXO 0748-676 in the table. Note however that the presence of an highly ionized plasma is nevertheless revealed by the detection of emission lines and absorption edges during the 
non-bursting emission of EXO 0748-676 (Cottam et al. 2001a; Jimenez-Garate et al. 2003), as is also true in the accretion disk corona source 4U 1822-371 (Cottam et al. 2001b). The most obvious property probably shared by the sources listed in Table 5 is to be viewed at high inclination. This condition is strengthened by the discovery of absorption lines in the dipping source XB 1916-053 reported here. Among the eight sources, only one, GX $13+1$, was suggested to have a low inclination, because it shows an unusually high radio to X-ray flux ratio, that can be explained by involving Doppler boosting of the radio emission, which implies that the radio jets point towards the observer and thus, that the source is seen close to poleon (Schnerr et al. 2003). All the other sources show strong evidence for being close to edge-on. This shows that inclination angle is important in determining the strength of these absorption features, which implies that the absorbing material is distributed in a cylindrical, rather than a spherical geometry, around the compact object. The azimuthal symmetry is implied by the lack of any orbital dependence of these features (e.g., Sidoli et al. 2001; Boirin \& Parmar 2003), apart during dipping, where the absorbing material seems to be less ionized (see below). Thus, the highly ionized material responsible for the absorption lines is likely to be related to the accretion disk. Such a highly ionized plasma may be common to systems accreting via a disk, but its observation made easy when the system is highly inclined.

Boirin \& Parmar (2003) noticed that the ratio of Fe XXVI (H-like) to FeXXV (He-like) line $E W \mathrm{~s}$ was higher in X 1254-690 than in MXB 1658-298 and GX 13+1, suggesting that the material responsible for the lines was more strongly ionized in X 1254-690. The authors proposed that the difference could be related to the overall size of the Roche lobe around the compact object into which the accretion disk must fit. Systems with shorter orbital periods are expected to have smaller accretion disks, and the obscuring material may be expected to be more photo-ionized in smaller systems with the same luminosities. As XB 1916-053 has the shortest orbital period and similar luminosities to the other systems (see Table 5), but has an absorbing material less strongly ionized than in X 1254-690, this hypothesis seems to be excluded.

Narrow absorption lines are detected in the EPIC pn persistent emission of XB 1916-053 at 6.65 and $6.95 \mathrm{keV}$ and identified with $\mathrm{K} \alpha$ resonant absorption lines from Fe XXV and Fe XXVI, respectively. Since both Fe XXV and Fe XXVI absorption features are detected, some physical parameters of the plasma responsible for the lines can be estimated. The column density of each ion can first be estimated from the $E W$ of the corresponding absorption line, using the relation quoted e.g., by Lee et al. (2002, see also references therein) linking the two quantities, which is valid if the line is unsaturated and on the linear part of the curve of growth, which is verified in the case of XB 1916-053. The ratio between Fe XXV and Fe XXVI column densities can then be used to estimate the photo-ionization parameter, $\xi$, using the calculations of Kallman \& Bautista (2001). Following this approach, we derive column densities of $3.4 \times 10^{17} \mathrm{~cm}^{-2}$ and $6.6 \times 10^{17} \mathrm{~cm}^{-2}$ for Fe XXV and Fe XXVI, respectively, and estimate $\xi$ to be $10^{3.92} \mathrm{erg} \mathrm{cm} \mathrm{s}^{-1}$ during the persistent emission. An absorption line consistent with Fe XXV K $\alpha$ is also detected in each intensity-selected dipping spectrum, and upper-limits have been set on the $E W$ of a Fe XXVI K $\alpha$ for each of these spectra. Thus, we also estimate $\xi$ to be $\lesssim 10^{3.83}$, $\lesssim 10^{3.68}$ and $\lesssim 10^{3.68} \mathrm{erg} \mathrm{cm} \mathrm{s}^{-1}$, during shallow, intermediate and deep dipping, respectively. These values are consistent with a decrease in ionization as dipping activity increases. This is again consistent with the presence of cooler material in the line of sight during dipping. We note that Parmar et al. (2002) also found evidence from the ratio of Fe XXV/Fe XXVI absorption line depths for the presence of additional cooler material in the line of sight in another dip source, X 1624-490, during dipping.

\section{Conclusion}

We have reported the discovery of narrow absorption lines at 6.65 and $6.95 \mathrm{keV}$, consistent with resonant Fe XXV and Fe XXVI K $\alpha$ transitions, in the persistent emission of the LMXB XB 1916-053. There is also marginal evidence for absorption features consistent with Mg XII, S XVI, Ni XXVII K $\alpha$ and Fe XXVI K $\beta$ transitions. Such absorption lines from highly ionized ions are now observed in a number of LMXBs seen close to edge-on $\left(i \sim 70^{\circ}\right)$, such as XB 1916-053. This suggests that the highly ionized plasma responsible for the absorption lines is related to the accretion disk. We have reported the dectection of the Fe XXV line in the dipping emission of XB 1916-053, and the upper-limits to the Fe XXVI column densities are consistent with a decrease in the amount of ionization during dipping intervals. This implies the presence of cooler material in the line of sight during dipping. We have also reported the discovery of a $0.98 \mathrm{keV}$ absorption edge in the persistent emission spectrum. The edge energy decreases to $0.87 \mathrm{keV}$ during deep dipping intervals. The detected feature may result from edges of moderately ionized $\mathrm{Ne}$ and/or Fe with the average ionization level decreasing from persistent emission to deep dipping. This is again consistent with the presence of cooler material in the line of sight during dipping.

Acknowledgements. Based on observations obtained with XMM-Newton, an ESA science mission with instruments and contributions directly funded by ESA member states and the USA (NASA). L. Boirin acknowledges an ESA Fellowship.

\section{References}

Asai, K., Dotani, T., Nagase, F., \& Mitsuda, K. 2000, ApJS, 131, 571 Becker, R. H., Smith, B. W., Swank, J. H., et al. 1977, ApJ, 216, L101 Bloser, P. F., Grindlay, J. E., Barret, D., \& Boirin, L. 2000, ApJ, 542, 989

Boirin, L., Barret, D., Olive, J. F., Bloser, P. F., \& Grindlay, J. E. 2000, A\&A, 361, 121

Boirin, L., \& Parmar, A. N. 2003, A\&A, 407, 1079

Brandt, W. N., Fabian, A. C., Dotani, T., et al. 1996, MNRAS, 283, 1071

Brandt, W. N., \& Schulz, N. S. 2000, ApJ, 544, L123

Callanan, P. J., Grindlay, J. E., \& Cool, A. M. 1995, PASJ, 47, 153

Chou, Y., Grindlay, J. E., \& Bloser, P. F. 2001, ApJ, 549, 1135

Church, M. J., Dotani, T., Balucinska-Church, M., et al. 1997, ApJ, 491, 388

Corbet, R. H. D. 2003, ApJ, 595, 1086 
Cottam, J., Kahn, S. M., Brinkman, A. C., den Herder, J. W., \& Erd, C. 2001a, A\&A, 365, L277

Cottam, J., Sako, M., Kahn, S. M., Paerels, F., \& Liedahl, D. A. 2001b, ApJ, 557, L101

Cottam, J., Paerels, F., \& Mendez, M. 2002, Nature, 420, 51

den Herder, J. W., Brinkman, A. C., Kahn, S. M., et al. 2001, A\&A, 365, L7

Frank, J., King, A. R., \& Lasota, J. P. 1987, A\&A, 178, 137

Galloway, D. K., Chakrabarty, D., Muno, M. P., \& Savov, P. 2001, ApJ, 549, L85

Grindlay, J. E., Bailyn, C. D., Cohn, H., et al. 1988, ApJ, 334, L25

Jansen, F., Lumb, D., Altieri, B., et al. 2001, A\&A, 365, L1

Jimenez-Garate, M. A., Schulz, N. S., \& Marshall, H. L. 2003, ApJ, 590,432

Kallman, T., \& Bautista, M. 2001, ApJS, 133, 221

Kallman, T. R., \& McCray, R. 1982, ApJS, 50, 263

Kaluzienski, L. J., Holt, S. S., Boldt, E. A., \& Serlemitsos, P. J. 1976, ApJ, 208, L71

Kotani, T., Ebisawa, K., Dotani, T., et al. 2000, ApJ, 539, 413

Kuulkers, E., Wijnands, R., Belloni, T., et al. 1998, ApJ, 494, 753

Lee, J. C., Reynolds, C. S., Remillard, R., et al. 2002, ApJ, 567, 1102

Miller, J. M., Wijnands, R., Méndez, M., et al. 2003, ApJ, 583, L99

Mirabel, I. F., \& Rodriguez, L. F. 1994, Nature, 371, 46

Morrison, R., \& McCammon, D. 1983, ApJ, 270, 119

Orosz, J. A., \& Bailyn, C. D. 1997, ApJ, 477, 876

Parmar, A. N., Oosterbroek, T., Boirin, L., \& Lumb, D. 2002, A\&A, 386,910
Parmar, A. N., White, N. E., Giommi, P., \& Gottwald, M. 1986, ApJ, 308, 199

Retter, A., Chou, Y., Bedding, T. R., \& Naylor, T. 2002, MNRAS, 330, L37

Ritter, H., \& Kolb, U. 2003, A\&A, 404, 301

Schmidtke, P. C. 1988, AJ, 95, 1528

Schnerr, R. S., Reerink, T., van der Klis, M., et al. 2003, A\&A, 406, 221

Shirey, R. E., Levine, A. M., \& Bradt, H. V. 1999, ApJ, 524, 1048

Sidoli, L., Oosterbroek, T., Parmar, A. N., Lumb, D., \& Erd, C. 2001, A\&A, 379, 540

Sidoli, L., Parmar, A. N., Oosterbroek, T., \& Lumb, D. 2002, A\&A, 385,940

Smale, A. P., Mason, K. O., White, N. E., \& Gottwald, M. 1988, MNRAS, 232, 647

Smale, A. P., Mukai, K., Williams, O. R., Jones, M. H., \& Corbet, R. H. D. 1992, ApJ, 400, 330

Strüder, L., Briel, U., Dennerl, K., et al. 2001, A\&A, 365, L18

Turner, M. J. L., Abbey, A., Arnaud, M., et al. 2001, A\&A, 365, L27

Ueda, Y., Asai, K., Yamaoka, K., Dotani, T., \& Inoue, H. 2001, ApJ, 556, L87

Ueda, Y., Inoue, H., Tanaka, Y., et al. 1998, ApJ, 492, 782

Walter, F. M., Mason, K. O., Clarke, J. T., et al. 1982, ApJ, 253, L67

White, N. E., \& Swank, J. H. 1982, ApJ, 253, L61

Yamaoka, K., Ueda, Y., Inoue, H., et al. 2001, PASJ, 53, 179

Yoshida, K., Inoue, H., Mitsuda, K., Dotani, T., \& Makino, F. 1995, PASJ, 47, 141 